Research Article

\title{
Multidimensional Development and $\pi$-Type Trend of the Blockchain Research: A Collaborative Network Analysis
}

\author{
Sun Meng $\mathbb{D},{ }^{1}$ Yan Chen $\mathbb{D}^{1},{ }^{1}$ Wei Zhou $\mathbb{D},{ }^{1}$ and Dejian Yu $\mathbb{D}^{2}$ \\ ${ }^{1}$ School of Finance, Yunnan University of Finance and Economics, Kunming 650051, China \\ ${ }^{2}$ Business School, Nanjing Audit University, Nanjing 211815, China \\ Correspondence should be addressed to Yan Chen; chenyan18@stu.ynufe.edu.cn
}

Received 25 November 2020; Revised 21 January 2021; Accepted 14 February 2021; Published 1 March 2021

Academic Editor: Mostafa M. A. Khater

Copyright (c) 2021 Sun Meng et al. This is an open access article distributed under the Creative Commons Attribution License, which permits unrestricted use, distribution, and reproduction in any medium, provided the original work is properly cited.

With the rapid growth of Internet technology, the blockchain has been developed fast in recent years with the wide expansions in finance, medicine, public welfare, and other fields. At the same time, the blockchain technology has also attracted researchers' increasing attention, leading to a large number of scientific outputs that made significant contributions to blockchain research. However, few attempts can be found to investigate the development of blockchain based on the literature in this area. Therefore, this paper focuses on blockchain research by introducing bibliometric methods and comprehensively analyzes its status quo, emerging trends, and development path. Based on the above research, this paper draws two main conclusions. First, we made a comprehensive investigation of blockchain research in the past five years and then found that the main research directions in this area are Bitcoin, digital currency, and Fintech. Second, the development of blockchain research generally presents a trend of $\pi$-transformation based on the main path analysis. The above studies provide a brief view for beginners who want to learn blockchain research in a direct way and contribute to extend the theoretical foundation of blockchain research by giving a comprehensive bibliometric analysis.

\section{Introduction}

As a new application of computer technology, blockchain has been widely used in recent years. Blockchain originated from Bitcoin and was first proposed by Satoshi Nakamoto in 2018. As a P2P virtual currency, Bitcoin can meet the demand of decentralization, strictly control the rate of money supply, estimate the total amount of money in circulation, and effectively suppress inflation. Bitcoin is basically a currency. It has some of the properties of a currency, but it is not necessarily a real currency. Unlike all currencies, Bitcoin is not issued by a specific monetary authority. It is created by a number of calculations based on a specific algorithm. The Bitcoin economy uses a distributed database composed of many nodes in the entire P2P network to confirm and record all transactions and uses cryptographic design to ensure the security of all aspects of currency circulation. The decentralized nature of $\mathrm{P} 2 \mathrm{P}$ and the algorithm itself ensure that the currency cannot be artificially manipulated by mass production of Bitcoins. The cryptographic-based design allows Bitcoins to be transferred or paid for only by their true owners. This also ensures the anonymity of the ownership of money and the transactions in circulation.

When Bitcoin was created, the term "blockchain" did not exist. Later, with the soaring price of Bitcoin and the stable operation of the Bitcoin system for many years, people began to care about the technology behind the Bitcoin system and develop the concept of "blockchain technology." Blockchain technology is also known as distributed ledger technology. It is a technical solution to collectively maintain reliable databases through decentralization and detrust. The technical scheme basically allows any number of nodes participating in the system to generate a series of data blocks related to the encryption method. Each data block contains all information of exchange data in the system during a certain period and generates a data fingerprint to verify the validity of its information and link to the next database block. Blockchain technology is the underlying technology of Bitcoin. It has a 
great mechanism and can be used in Bitcoin or many other fields.

The research and application on blockchain mainly focus on the following aspects. The first is digital cryptocurrency. The widespread use of Bitcoin has proved the feasibility and great potential of issuing digital currency based on blockchain technology. Harvey [1] discussed the operation mechanism of Bitcoin in detail and pointed out that Bitcoin was a successful application of cryptofinance in digital cryptocurrency. With the help of an encryption algorithm, ownership authentication and transaction can be realized under the condition of decentralization. The second is financial trading and clearing. Lee [2] sorted out the operation mechanism of blockchain technology. This research focused on the legislation and regulation of the point-to-point blockchain trading market and analyzed the impact of blockchain technology on naked short selling and highfrequency trading. It is derived that blockchain technology is easy to use in the exchange transaction settlement which could negatively influence transaction behaviors. The third is the smart contract. Christidis and Devetsikiotis [3] argued that smart contracts can organically combine blockchain with Internet of Things (IoTs) technology. Blockchain technology entrusts each isolated smart device with a trust mechanism and thus creates a service trading market among smart devices. In this sense, it promotes the application and popularization of IoT technology. However, there are still problems such as user privacy protection and legal authorization. The last is corporate governance. Because blockchain technology is suitable for the company equity trading and ownership registration, Yermack [4] discussed that all participants are involved in corporate governance because of the blockchain. Hence, if this technology is applied in the equity of listed enterprises, all shareholders' shareholdings will be completely transparent and traceable. It leads to the exposure of Insider-Trading and Back-Dating. Therefore, blockchain technology could positively impact corporate governance.

With the deepening research on blockchain, it is particularly important to understand the status quo and development trend of the field. Based on the literature data about blockchain obtained from Web of Science, this paper analyzes the status quo and development trend of blockchain research with the most popular bibliometric software, CiteSpace, which involves the process of knowledge mining, analysis, organization, mapping, and display. Yu [5] made a visual analysis of aggregation operators by using CiteSpace. According to the research results, the main research clusters and their corresponding key elements in this region were revealed, and the close relationships among major journals and important authors could be found. To determine the current situation and research trends in the public-private partnership field, Song et al. [6] conducted a series of content analyses using the CiteSpace software package and drew three important conclusions. Chen and $\mathrm{Wu}$ [7] investigated the literature on makers from 1975 to 2016 and performed the visual analysis with CiteSpace bibliometrics software to identify major intelligent cooperative networks, cooccurrence keywords, research clusters, and iconic articles. Cui and Zhang [8] made a comparative study of public policies and research on the development of circular economy in China by using literature measurement software CiteSpace, and the research results showed that there was obvious interactive feedback between public policies and academic research. Olmeda-Gómez et al. [9] used CiteSpace to conduct a qualitative and visual analysis of epigenetic literature published between 2009 and 2017 to gain an in-depth understanding of patterns and trends in the field and to identify innovative and potential transformational studies. Even though blockchain is a concept that has emerged in recent years, the bibliometric studies about blockchain research are seldom found. Therefore, CiteSpace was used in this paper to analyze the emerging trends and current status in blockchain research, including cocitation analysis of authors, institutions, countries, and journals, as well as burst detection of keywords to further analyze the emerging trends and hotspots in blockchain research.

In addition, to fully understand the development path of blockchain research, this paper also introduces the main path analysis. Citation network analysis is an effective tool, which can reflect the influence of a paper on the subject. Main path analysis is one of the most concerns in recent years. Compared with the commonly used $\mathrm{H}$-index and G-index, it not only considers the direct impact but also considers the indirect impact. Moreover, the main path analysis can correct the problem of citation bias caused by different publication years. Because in this approach, the latest paper is not compared with the time-honored papers, but with papers published at the same time. When conducting author cocitation analysis, Ma et al. [10] used the social network analysis tool Pajek to visualize the authors' cocitation matrix and finally pointed out the similarities and differences between Chinese information science and foreign information science. Kozak et al. [11] used bibliometric software Pajek and VOSviewer to study international collaborations between researchers affiliated with eastern European countries in 1999, 2000, and 2001. Therefore, based on CiteSpace and Pajek, this paper makes a rounded analysis of the status quo, emerging trend, and development path of blockchain research.

In this paper, there are several obvious advantages to other research. First, the scope of this study focuses on the blockchain field. Besides, cluster analysis and author collaboration networks are used to illustrate the links between articles and authors and to display papers with strong citation bursts at different time intervals, and burst detection is introduced in this paper. Finally, this paper provides a detailed overview of the development of blockchain research by using four different main path analyses to provide a more comprehensive understanding of the development of blockchain research. Thus, this study aims to provide a more objective and comprehensive econometric analysis of the literature on blockchain research through the use of software CiteSpace and Pajek. Based on the following aspects, 1230 published studies on blockchain research retrieved from Web of Science (WoS) were analyzed in this paper: (1) analyze the authors who have had a significant impact on the field; (2) state the countries/regions, periodicals, and 
institutions that have made significant contributions; (3) identify the emerging trends and current situation of blockchain research; and (4) understand the development path of blockchain research.

The article structure of the paper is mainly as follows. Section 2 focuses on the analysis of citations. Section 3 provides a description and analysis of the countries/regions, institutions, and authors that have published a large number of studies on the blockchain. Section 4 describes the emerging trends and hotspots in the field using burst detection techniques and also presents the results of four different main path analyses. Finally, Section 5 gives the main findings and conclusions.

\section{Methodology}

CiteSpace, short for Citation Space, is a tool to analyze the potential knowledge embedded in the scientific literature. It also provides a comprehensive overview of specific research fields in terms of scientometrics, data, and information visualization. It is a multifaceted, time-dependent, and dynamic citation visualization tool that was developed in the context of data and information visualization. We can also call this method "Mapping knowledge domains" because the structure, pattern, and distribution of scientific knowledge are presented through visualization. Compared with other bibliometric software, CiteSpace can perform clustering analysis on data from different aspects, making the results more reliable. Also, CiteSpace has the following advantages: (1) it can process large amounts of text data, making the analysis more extensive, and it can also change the format of the data so that the data set can be applied to other software; (2) information visualization can be realized to make the analysis results more intuitive and specific; (3) CiteSpace can also perform burst analysis and trend analysis.

Pajek was originally developed by Vladimir in 1996. As an analysis tool for large complex networks, Pajek is a powerful tool for studying existing complex nonlinear networks with thousands or even millions of nodes. Compared with network analysis tools such as Ucinet and Gephi, Pajek can process more nodes and links quickly and support the analysis of massive data. Specifically, there are three characteristics that Pajek has: (1) the algorithm is fast and effective. All the algorithms of Pajek are less than order 2. Therefore, it is very effective for the calculation of complex networks. (2) Pajek provides a visual operation platform for users with its humanized interface and simple operation. (3) Users can not only use Pajek to find out the local characteristics of the complex network but also view the structure of the whole network from a global perspective.

Building CiteSpace and Pajek analysis databases require two steps. The first step is to select the database. We choose Web of Science as the database because it is a huge platform for providing readers with extensive and peer-reviewed research outputs published on Social Science Citation Index (SSCI), Science Citation Index Extension (SCI-E), and other databases. The second step would be the selection of appropriate keywords. Researchers should pay more attention to the validity and representativeness of keywords because they can largely determine the accuracy of research fields. In this paper, a total of 1,230 articles published between 2015 and 2019 have been retained in the blockchain research, excluding the citation index of conference process-science. The research time of this study was selected for two reasons: (1) a large number of papers with significant contributions have been published since 2015; (2) this study is related to the latest research on blockchain, and it is regarded as the latest paper in this research field.

\section{Cluster Network and the Most Cited Articles}

According to the steps above, this paper downloaded the literature about blockchain research via Web of Science on November 1,2019, based on 1,230 papers published between 2015 and 2019.

To establish a better professional development mechanism, the concept of cocitation and cluster is introduced. If two papers are cited by one or more subsequent papers at the same time, the two papers are said to be cocitation. Cluster analysis divides all literature into several different categories according to a certain property. Cocitation and cluster analysis of blockchain research can be obtained by using CiteSpace, as shown in Figure 1 and Table 1. Cocited articles are grouped into clusters to make the main research areas easier for readers to understand. Table 2 shows the detailed results of the main cocited articles.

Figure 1 shows the cluster network in the blockchain research of 1230 pieces of literature. As can be seen from Figure 1, there are 9 clusters, trust degree, distributed ledger technology, tutorial, healthcare, narrative, trust, consensus algorithm, and supply chain management, respectively. Table 1 shows the top 7 clusters in the blockchain research field. The size represents the number of references contained in each cluster. The log-likelihood ratio (LLR) is the algorithm that calculates each label and summarizes each cluster. In addition, the homogeneity of the clusters is tested by the silhouette score. The range of the silhouette is 0 to 1 . When the silhouette is greater than 0.5 , the corresponding result is considered to be reliable. The closer the silhouette is to 1 , the more reliable the result is. As can be seen from Table 1, all silhouette scores are above 0.7 , indicating that the quality of each cluster is reliable. The largest cluster is \#0 cycle tours, with 31 member references. In addition, the average year for most clusters is around 2015.

Based on the understanding of the top 7 clusters in the blockchain research field, the top 20 articles with more than 40 times of cocitation frequency and their clusters are shown in Table 2. Chen et al. [31] believed that the most cited papers made significant contributions to a certain research field. It is clear to see that the most cited articles are almost from \#1 and \#3. Therefore, it can be seen from Table 2 that cluster 3 is the most cited cluster being cited 186 times. This article is "Blockchain and smart contracts for the IoT." Furthermore, the article "Blockchain thinking the brain as a decentralized autonomous corporation" is from \# 1 with both 132 citations, and the article "Bitcoin and beyond: A technology survey on decentralized digital currencies" is from \#0 with both 83 citations. 


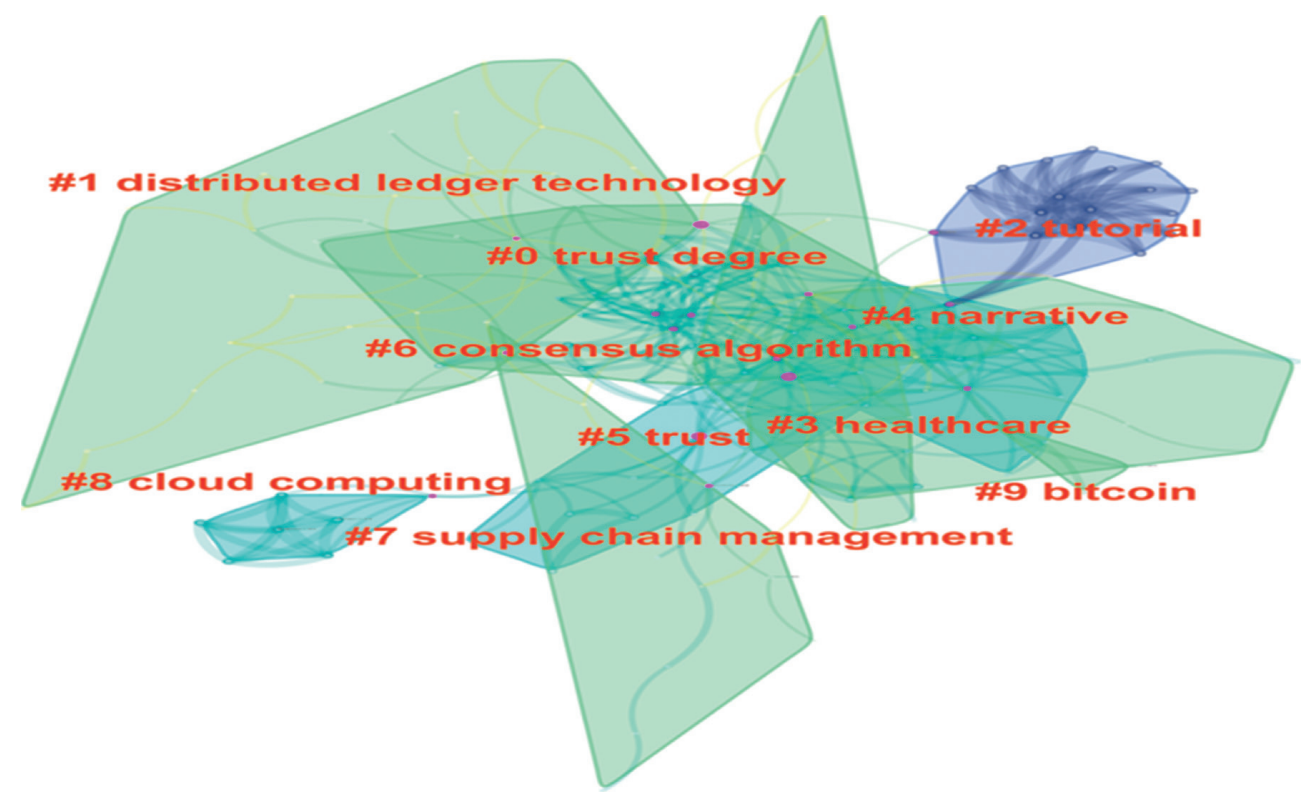

FIGURE 1: Cluster network in the blockchain research.

TABle 1: The largest 7 clusters in the blockchain research.

\begin{tabular}{lcccc}
\hline $\begin{array}{l}\text { Cluster- } \\
\text { ID }\end{array}$ & Size & Silhouette & Label (LLR) & $\begin{array}{c}\text { Mean } \\
\text { (year) }\end{array}$ \\
\hline 0 & 31 & 0.706 & $\begin{array}{c}\text { Trust degree } \\
\text { Distributed ledger } \\
\text { technology }\end{array}$ & 2015 \\
1 & 29 & 0.840 & Tutorial & 2016 \\
2 & 18 & 0.966 & Healthcare & 2016 \\
3 & 18 & 0.730 & Narrative & 2015 \\
4 & 16 & 0.758 & Trust & 2014 \\
5 & 13 & 0.762 & Consensus algorithm & 2016 \\
6 & 12 & 0.834 & $\begin{array}{c}\text { Supply chain } \\
\text { management }\end{array}$ & 2016 \\
7 & 11 & 0.873 & &
\end{tabular}

\section{Contribution Analysis}

Not only that but also the number of results is also correlated with research contribution. This part analyzes their contributions to the research field of blockchain from several aspects, including countries, institutions, journals, and authors. The results show that the most productive countries are also in the boom period of blockchain research, and the most productive institutions are mainly from these countries. In addition, papers by prolific authors are highly influential in blockchain research.

4.1. Analysis of Countries and Institutions. To make the results more reliable and objective, this paper used WoS records to analyze the countries with the highest generation efficiency. Besides, CiteSpace was used to analyze the most cited authors and collaborative networks among institutions. A detailed analysis of the results is given below.

Table 3 shows the top 10 countries with the research literature on the blockchain. It can be seen from the table that China is the country with the largest contribution,
TABle 2: Top 20 most cited articles in the blockchain research.

\begin{tabular}{lcc}
\hline $\begin{array}{l}\text { Citation } \\
\text { counts }\end{array}$ & References & Cluster number \\
\hline 186 & Christidis and Devetsikiotis [3] & 3 \\
132 & Swan [12] & 1 \\
105 & Zyskind and Nathan [13] & 3 \\
83 & Tschorsch and Scheuermann & 0 \\
73 & [14] & 1 \\
72 & Yue et al. [15] & 1 \\
69 & Azaria et al. [16] & 1 \\
67 & Yli-Huumo et al. [17] & 0 \\
65 & Kosba et al. [18] & 0 \\
62 & Zheng et al. [19] & 2 \\
57 & Aitzhan and Svetinovic [20] & 3 \\
54 & Dorri et al. [21] & 3 \\
54 & Kang et al. [22] & 1 \\
53 & Kuo et al. [23] & 1 \\
52 & Underwood [24] & 0 \\
50 & Wood [25] & 1 \\
50 & Tapscott and Tapscott [26] & 3 \\
50 & Li et al. [27] & 3 \\
49 & Khan and Salah [28] & 0 \\
48 & Eyal [29] & 3 \\
\hline
\end{tabular}

ranking first with 420 papers published, accounting for $34 \%$. USA, England, and South Korea followed with more than $40 \%$. Although relatively few papers have been published from other countries, their contributions to this field of research have also been substantial.

Figure 2 shows a visual analysis of the national collaborative network for blockchain research. The node sizes in the figure represent the number of publications by country and correspond to Table 3. It can be found that there is certain geographic cooperation between these countries. High-yielding countries in the field of blockchain research cooperate more with other countries, while low-yielding countries cooperate less. 


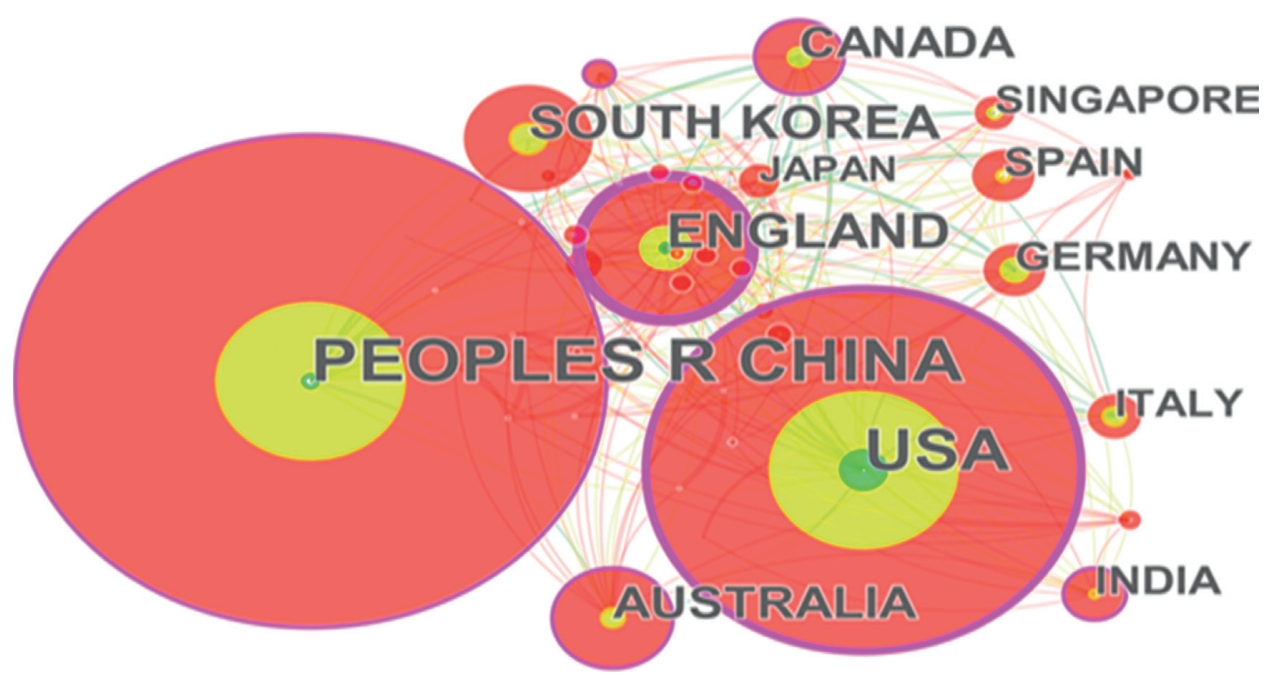

FIgURE 2: A visualization of the country collaboration network in blockchain research.

In addition, we can find that the number of published papers is related to the number of research institutions and the amount of research funds. As shown in Table 4, among the top 10 institutions that have made significant contributions to 156 institutions that have published research on blockchain, the Beijing University of Posts and Telecommunications is in a prominent position in this field, with 41 papers published. The rest institutions are mostly from China. Nine of the 10 institutions that made significant contributions came from China, accounting for about 15\% of the total number of papers published in the area. As mentioned above, China is the country with the most published papers in blockchain research, so it can be concluded that the most productive institutions come from the most productive countries. China is unquestionably the most productive country in the field.

Furthermore, Figure 3 shows the global collaborative network of research institutions on blockchain research from 2015 to 2019. The network structure can be seen from the maturity of cooperation between institutions. If the structure is close enough, the cooperation between corresponding institutions will be relatively mature. As can be seen from Figure 3, there are more cooperation between institutions in the same country. Therefore, it is essential to strengthen international interaction, especially those countries that have a significant impact on the development of blockchain research.

4.2. Analysis of Journals and Authors. Table 5 is the top 10 journals that have published research literature on the blockchain, and these data are from WoS. It can be seen from the table that IEEE Access is the journal with the most published literature, with 189 articles published in total, accounting for $15.37 \%$ of all publications, and it has made great contributions to the blockchain research field and has a large number of research results. This is followed by the IEEE Internet of Things Journal, Sensors, and Applied Sciences Basel, which together have published more than 100 papers related to blockchain research.
TABle 3: Top 10 productive countries in the blockchain research.

\begin{tabular}{lcc}
\hline Countries/regions & Number & Percentage \\
\hline China & 420 & 34.14 \\
USA & 309 & 25.12 \\
England & 116 & 9.43 \\
South Korea & 93 & 7.56 \\
Australia & 84 & 6.83 \\
Canada & 65 & 5.28 \\
Germany & 48 & 3.96 \\
Spain & 45 & 3.66 \\
India & 42 & 3.41 \\
Italy & 40 & 3.30 \\
\hline
\end{tabular}

Not only that but also articles in the lower ranked journals have made a huge contribution to the development of the field, such as "A secure fabric blockchain-based data transmission technique for industrial IoT" published by IEEE Transactions on Industrial Informatics and "On blockchain and its integration with loT-Challenges and opportunities" published by Future Generation Computer Systems, The International Journal of eScience. These articles are also often cited when dealing with the preferences and uncertainties of decision makers to improve further investigation. To sum up, both the journals listed in Table 5 and their published articles are of great significance to the advancement of blockchain research.

Table 6 lists the top 10 productive authors. According to WoS records, Kim-Kwang and Jong are the most prolific authors of blockchain research, with 16 and 14 papers, respectively. Of particular note, the above 16 and 14 papers are not the actual research output of each author because some papers were filtered out according to the search method in this study, but the influential papers of these authors are generally taken into account. In terms of affiliations, Kim-Kwang is the professor of the Queensland University of Technology and Jong is the professor of the Department of Computer Science and Engineering, Seoul National University of Science and Technology. Figure 4 is a collaborative network diagram of the main authors of the blockchain study. The figure confirms the conclusion of 
TABLE 4: Top 10 productive institutions in blockchain research.

\begin{tabular}{lcc}
\hline Institutions & Number of publications & Percentage \\
\hline Beijing University of Posts and Telecommunications & 41 & 3.33 \\
Xidian University & 22 & 1.79 \\
University of Science and Technology of China & 21 \\
The University of Texas at San Antonio & 20 \\
Chinese Academy of Sciences & 18 \\
Beijing Institute of technology & 16 \\
Guangdong University of Technology & 15 \\
Wuhan University & 15 \\
Beihang University & 14 \\
Nanyang Technological University & 14 \\
\hline
\end{tabular}

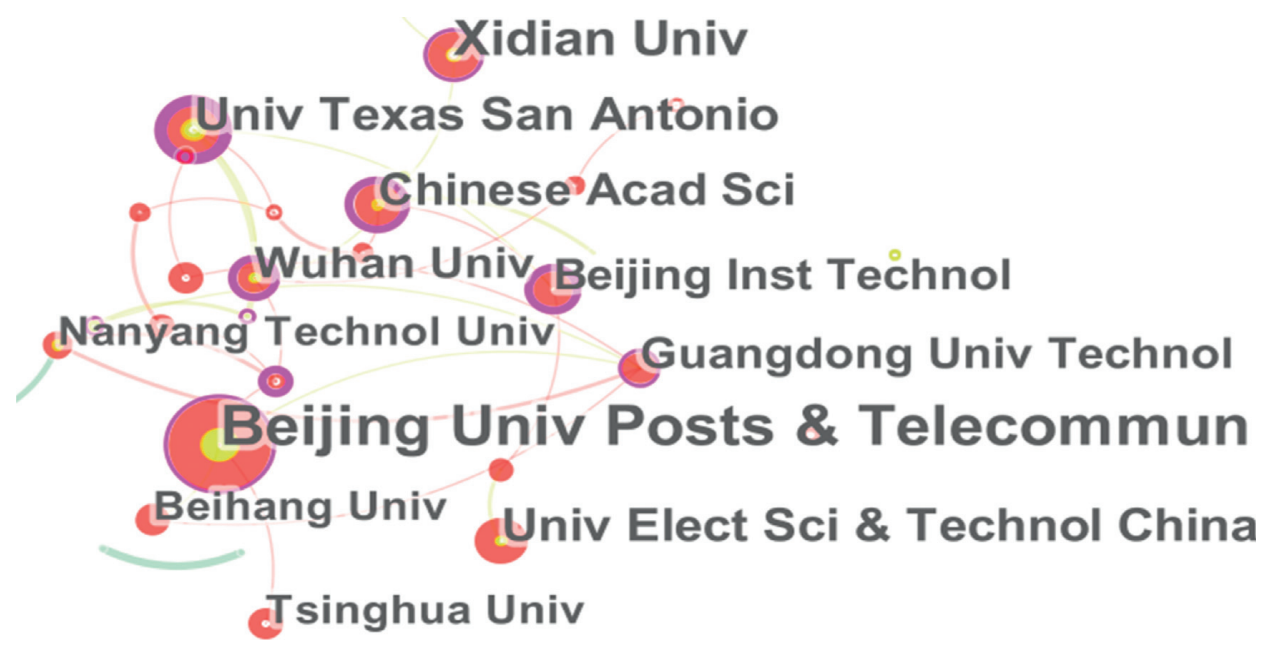

FIgURE 3: A visualization of the institution collaboration network in blockchain research.

TABLE 5: Top 10 productive journals in blockchain research.

\begin{tabular}{lcc}
\hline Journals & Number of publications & Percentage \\
\hline IEEE Access & 189 & 15.37 \\
IEEE Internet of Things Journal & 44 & 3.58 \\
Sensors & 34 & 2.76 \\
Applied Sciences Basel & 25 & 2.03 \\
IEEE Transactions on Industrial Informatics & 22 & 2.03 \\
Future Generation Computer Systems, The International Journal of eScience & 22 & 1.79 \\
Sustainability & 21 & 1.79 \\
IT Professional & 19 & 1.70 \\
Energies & 17 & 1.54 \\
Journal of Medical Systems & 1.38 \\
\hline
\end{tabular}

Table 6 that Kim-Kwang and Jong are prolific authors in this field. Besides, it can be found that Kim-Kwang and Jong not only have a lot of cooperation but also cooperate with scholars such as Xiao, Mawson, and Liehuang, who are also the productive authors listed in Table 6.

To further investigate authors who have had a significant impact on blockchain research, we made an author cocitation network, as shown in Figure 5, which shows authors who have been continually cited in recent years. It is important to note that this study only considers the first author of a given paper.

The most cited authors are Nakamoto, Christidis, and Swan. Blockchain originated from Bitcoin, and Nakamoto published the paper "Bitcoin, a peer-to-peer electronic cash system," which marked the birth of Bitcoin. Since Nakamoto's theory of Bitcoin, various approaches using P2P technology, cryptography, timestamping, blockchain technology, and other electronic cash system architecture concepts have been developed. As Nakamoto has provided a crucial theoretical foundation for blockchain research, he has become one of the most renowned scholars in the field of blockchain.

The comparison between Table 5 and Figure 5 shows that there is a certain connection between the two. The prolific authors and the frequently cited authors are basically the same, but there are still some differences. 
TABLE 6: Top 10 productive journals in blockchain research.

\begin{tabular}{lcc}
\hline Journals & Number of publications & Percentage \\
\hline IEEE Access & 189 & 15.37 \\
IEEE Internet of Things Journal & 34 & 3.58 \\
Sensors & 25 & 2.76 \\
Applied Sciences Basel & 25 & 2.03 \\
IEEE Transactions on Industrial Informatics & 22 & 1.03 \\
Future Generation Computer Systems, The International Journal of eScience & 22 & 1.79 \\
Sustainability & 21 & 1.70 \\
IT Professional & 19 & 1.54 \\
Energies & 17 & 1.38 \\
Journal of Medical Systems & 17 \\
\hline
\end{tabular}

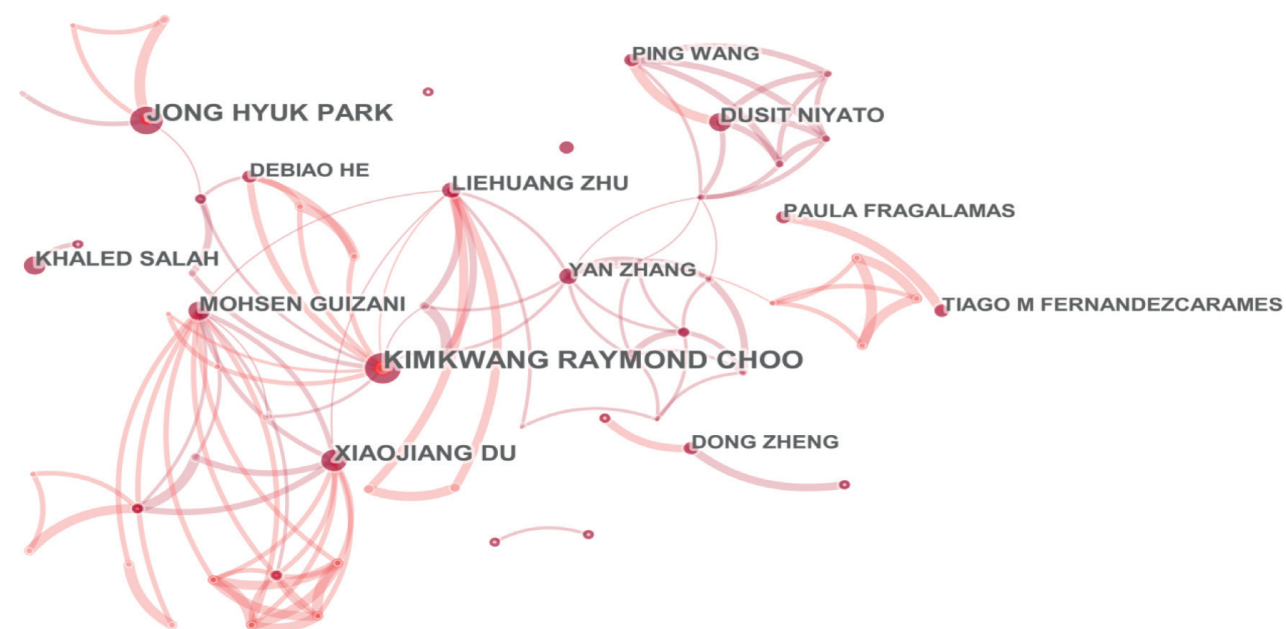

FIGURE 4: Collaborative network diagram for blockchain research.

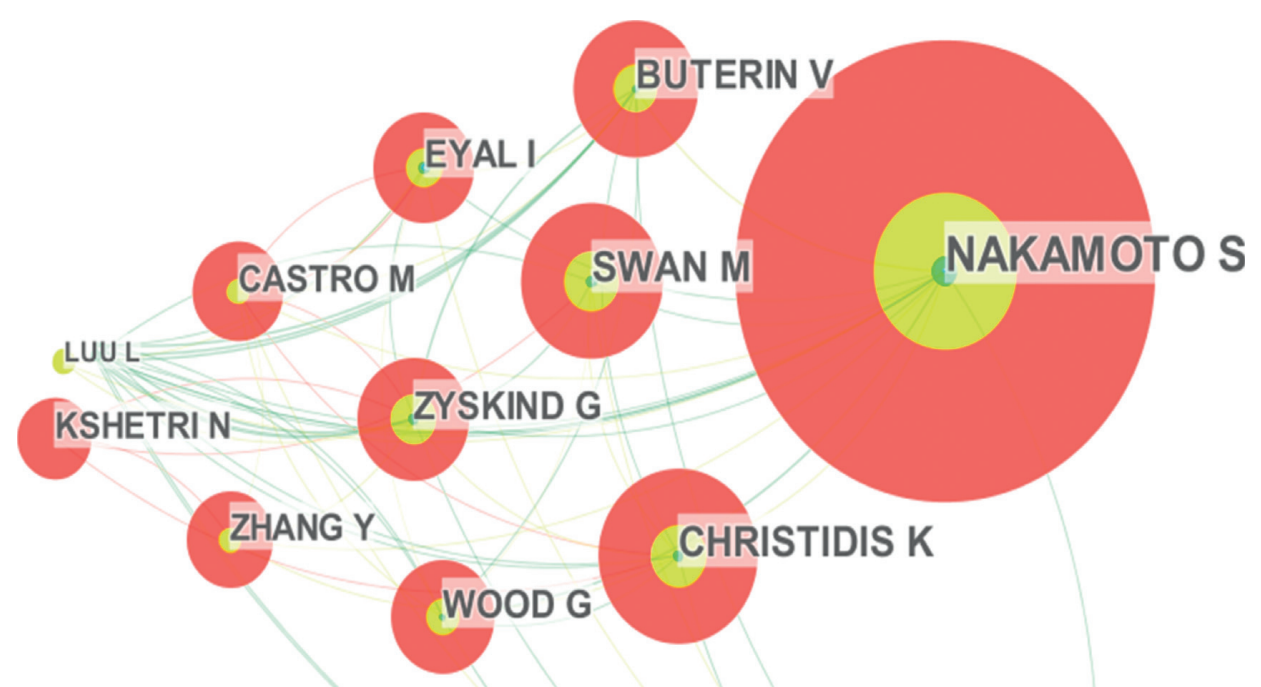

FIGURE 5: A visualization of the author cocitation network in blockchain research.

As more and more scholars are paying attention to blockchain and its rapid development, it is of great significance to discuss this issue from different perspectives. To clearly show the hotspots in different years and the evolution process, we have conducted a selection process of research directions. Table 7 lists the top 10 research directions in this field. As can be seen, "Computer Science" ranks first with 666 articles, followed by "Engineering" and "Telecommunications." The first three directions are the hotspots in the blockchain research field. Besides, emergencies still exist, which is a new direction for this research field. More information can be found below. 
TABLE 7: Top 10 research directions in blockchain research.

\begin{tabular}{lcc}
\hline Research direction & Number of publications & Percentage (\%) \\
\hline Computer Science & 666 & 54.15 \\
Engineering & 505 & 41.06 \\
Telecommunications & 407 & 33.09 \\
Business Economics & 125 & 10.16 \\
Science Technology and other topics & 74 & 6.02 \\
Chemistry & 65 & 5.28 \\
Healthcare Sciences Services & 45 & 3.66 \\
Environment Sciences Ecology & 40 & 3.25 \\
Instruments Instrumentation & 39 & 3.17 \\
Medical Informatics & 39 & 3.17 \\
\hline
\end{tabular}

\section{Emerging Trends and Development Context Analysis}

5.1. Emerging Trends. Scholars also focus on emerging trends when researching blockchain, and they shed light on new research directions and cutting-edge methods. Citation frequency is a measure of the recognition of a research paper by other institutions or scholars. Burst detection can identify the influential events occurring in a certain period of time and can be used to detect the center and focus of research in a certain period of time in bibliometrics. In this section, the burst detection of references, keywords, and cited authors are used to illustrate the articles in blockchain research that gets a fast growth of citations and focuses on exploring research directions.

The rapidly increasing number of citations can reflect emerging trends in a particular field of research. The increase in citation frequency indicates that scholars pay more attention to the corresponding publications. In this paper, the reference burst detection is used to understand the emerging trends of blockchain research. As shown in Figure 6, from 2015 to 2019, there were 10 papers with high citation frequency. It can be seen that all the citation explosions occurred after 2015, and the earliest citation explosion in this research field occurred in 2016, which is consistent with the rapid development stage (2016-2019) of the blockchain research mentioned above. From 2016 to 2019, researchers focused on blockchain and smart contracts for the IoT [3], cooperate governance and blockchains [4], blockchain technology: principles and applications [32], and blockchain: the state of the art and future trends [33].

Considering the sudden detection of keywords, this paper gives topics that are rapidly developing in this research area. Figure 7 lists the top six keywords with bursts. The keyword "Bitcoin" was proposed in the blockchain for the first time. The "digital currency" was introduced in 2017 and has attracted much attention from 2017 to 2019. Therefore, the outbreak time of this keyword is from 2016 to 2019. Some topics show that research topics change rapidly over time.

From Figure 8, all the authors received the citation bursts in 2016. Merkle and Antonopoulos, as cited authors, have the longest duration that began in 2016 and ended in 2019 years. Moreover, the strength of Gervais is the strongest.

5.2. Development Context Analysis. To further understand the development path of blockchain research, this paper uses Pajek to analyze the main path of blockchain research. Four basic paths summarize the development of blockchain research from different perspectives.

5.2.1. Local Forward Main Path. A total of 9 papers appear to the local forward main path. The path in Figure 9 follows the most path generation method, which demonstrates the knowledge diffusion in a specific domain and helps researchers capture the knowledge backbone to understand blockchain research comprehensively.

As one of the earliest articles to study blockchain [3], this paper examined whether they make a good fit for the IoT sector. Through the study of blockchain and IoT, Christidis concluded that the combination of blockchain and the Internet of Things is powerful and can bring about significant changes in multiple industries, pointing the way for new business models and distributed application. Ali et al. [34] also talk about the combination of blockchain and the IoT; based on the challenges posed by the current centralized Internet of Things model and combined with the latest advances in industry and research, this paper effectively uses the blockchain to provide a decentralized and secure medium for the Internet of Things.

Tschorsch and Scheuermann [14], also as one of the earliest articles, discussed the impact of blockchain in the field. In this paper, they got a variety of research results and research directions. They continued to explore the design space by discussing existing contributions and results, based on an introduction to the Bitcoin protocol and its building blocks. Finally, based on the above discussion, they came to the core basic structure and insights into Bitcoin protocol and its application. As the paper showed and discussed, many of the key ideas apply to every other field, so their impact goes well beyond Bitcoin itself.

As shown in Figure 9, another earliest paper is by Kraft [35]. This paper used a Poisson process model with timedependent intensity to derive block-time predictions for various hash rate scenarios and also analyzed the method of updating "network difficulty" as a mechanism to keep blocktime stable. Next, Zheng et al. [19] introduced the classification and consensus algorithm of blockchain, reviewed the application of blockchain, discussed the technical challenges and the latest progress in addressing them, and pointed out the development direction of blockchain technology. Li [36] proposed a secure energy trading system called energy 


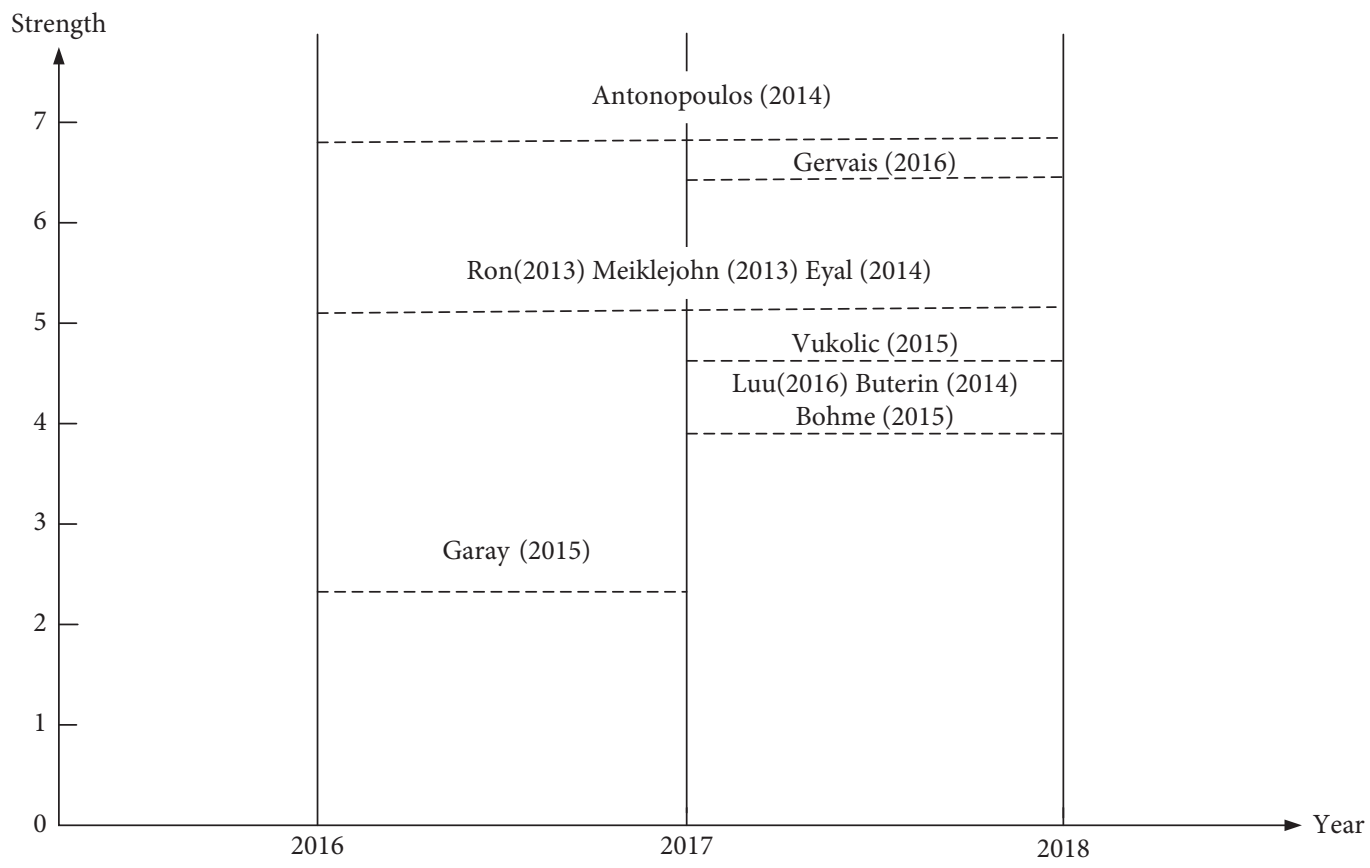

Figure 6: Top 10 references with strongest citation bursts in blockchain research.

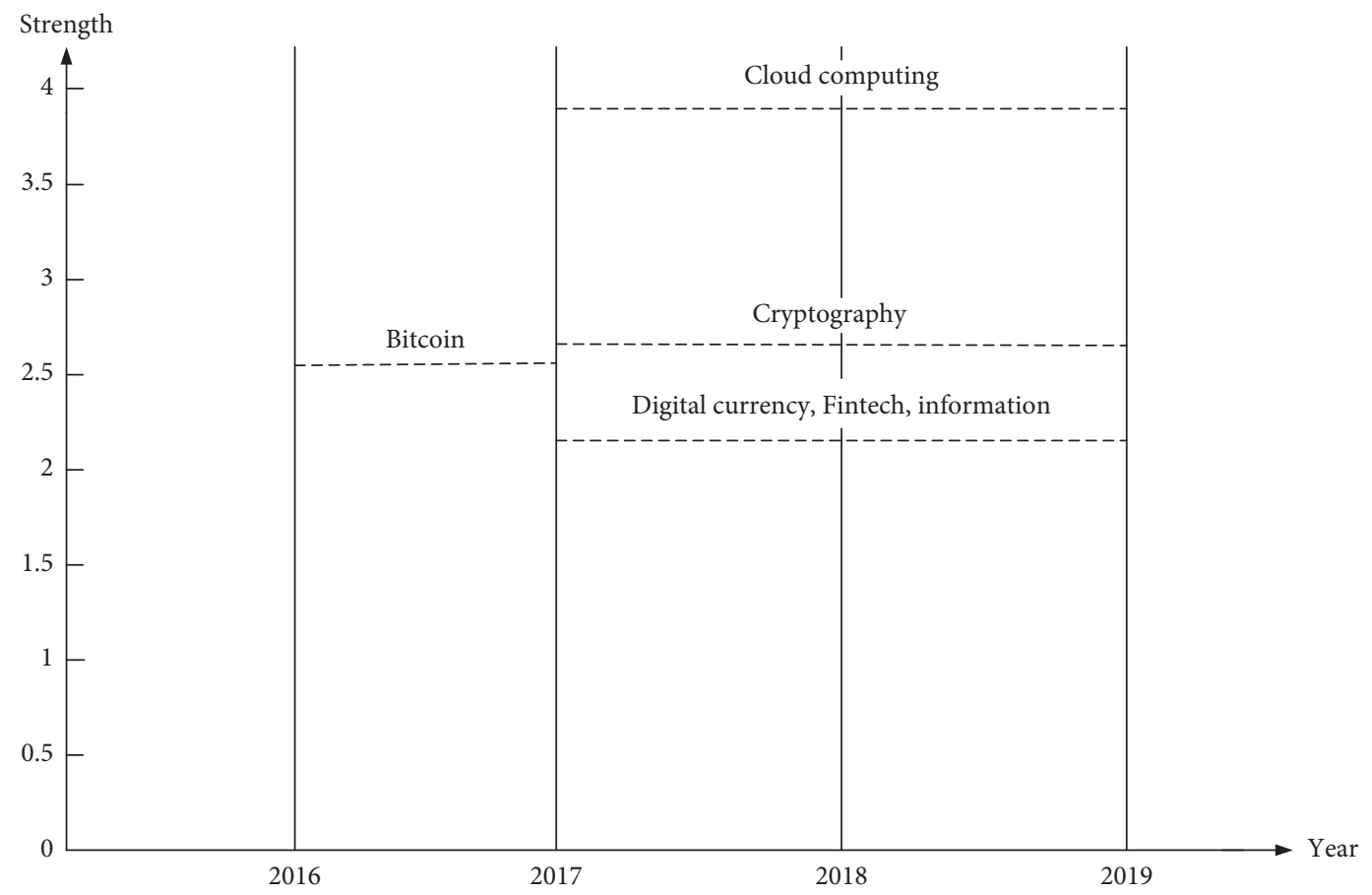

FIGURE 7: Top 6 keywords with strongest citation bursts in blockchain research.

blockchain by using blockchain technology of consortium. This kind of energy blockchain can be widely used in general $\mathrm{P} 2 \mathrm{P}$ energy trading scenarios, to get rid of the trusted intermediary. Kang et al. [37] realized the secure data storage and sharing of vehicle edge network by using the blockchain of consortium and smart contract technologies.

Based on blockchain, Nizamuddin et al. [38] proposed a document sharing and version control solution and framework to facilitate multiuser collaboration, without involving centralized trusted entities or third parties, and to track changes in a trusted, secure, and decentralized manner. As the final paper, a new blockchain-based incentive anonymous reporting system, ReportCoin, was proposed in 2019 (Zou et al.). ReportCoin guarantees user identity privacy and the reliability of reporting messages throughout the reporting process. 


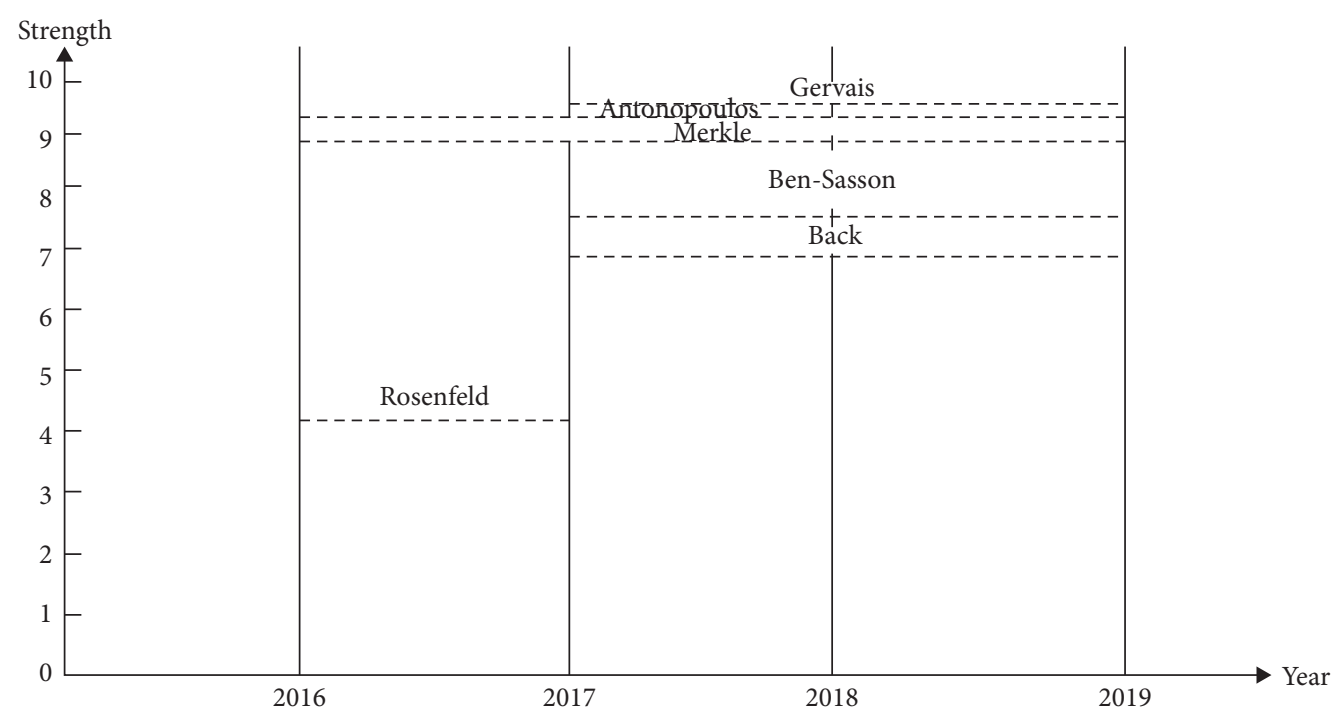

FIGURE 8: Top 6 cited authors with the strongest citation bursts in blockchain research.

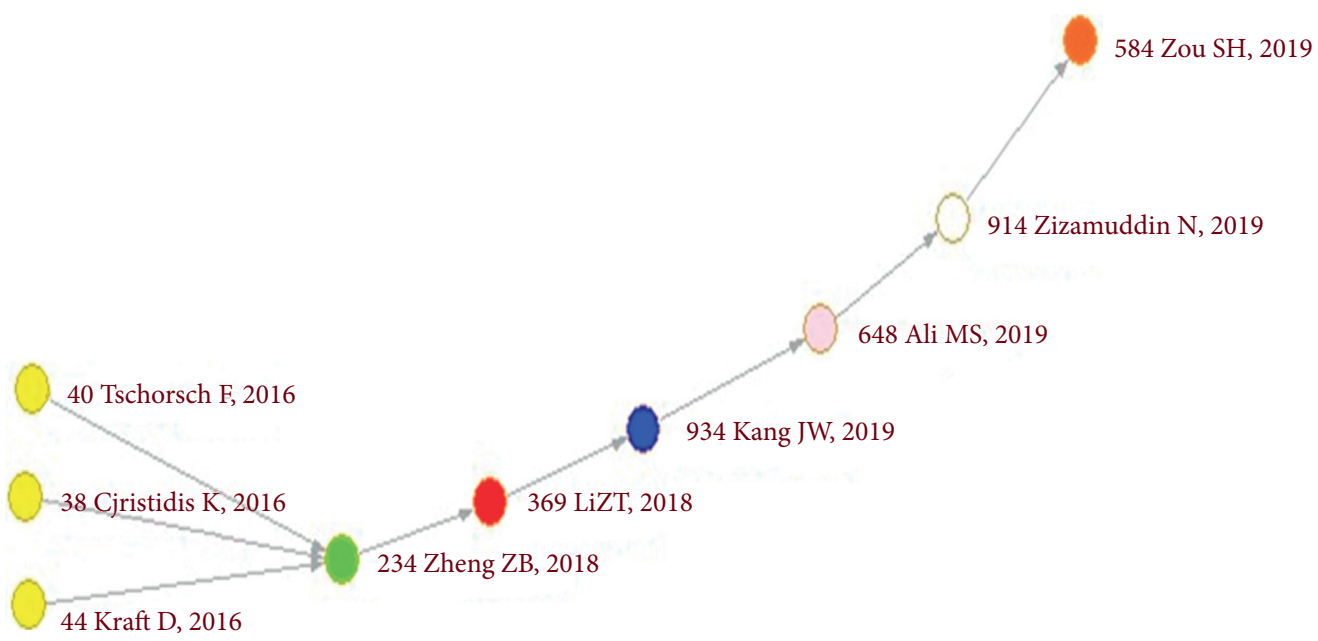

FIGURE 9: Local forward main path analysis of blockchain research.

Due to the number of papers that can be shown in the main path, some important papers are omitted in Figure 9. Therefore, we comply with the view of Liu and Lu [39] which suggested more than one path was identified from different perspectives. Some influential articles will be given in the following path analysis.

5.2.2. Local Backward Main Path. The local backward main path in Figure 10 is opposite to the local forward main path, which tracks development trajectories from now to before. It pays more attention to the works in the latest years. Therefore, it is not surprising that the works in Figure 10 are newer than Figure 9. After a detailed inspection, it is found that most of them have received considerable recognition, which can be seen from the cited score.

From Figure 10, we can see that the local backward main path has two branches. The first path deals with the application of blockchain in the IoT, game theory, mobile services, and other fields. Like the first paper, Kim [40] combined blockchain with game theory, and this paper proposes a new model that enables existing blockchain control schemes to be applied directly to real-world operations. Besides, Liu [41] also studies game theory and blockchain technology, and this paper fills in the gaps in the current research on the blockchain network, in which game theory is used as an analysis tool and in which there is a lack of comprehensive investigation on the application of game theory methods in blockchain-related issues. In the following, in order to compensate for the literature review on the development of decentralized consensus mechanisms in blockchain networks, Wang et al. [42] provided a systematic view of the organization of blockchain networks. In the next two papers, Liu et al. [43] and Xiong et al. [44] both introduced the application of blockchain in the wireless mobile network. They put forward moving edge calculation to solve 


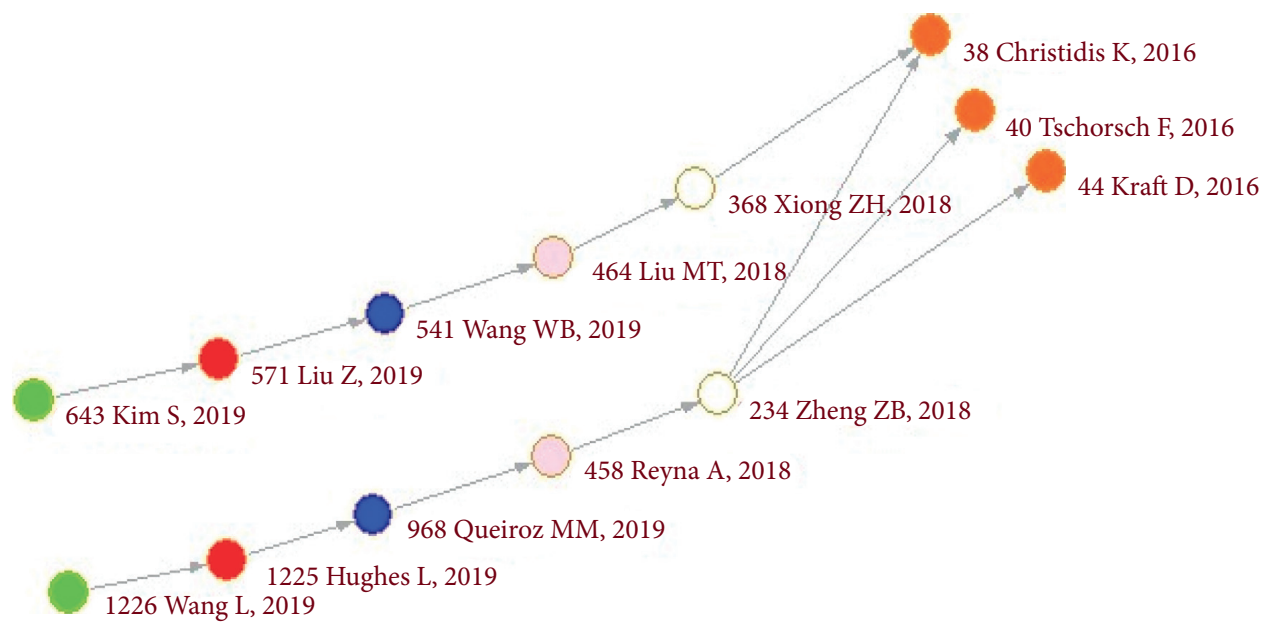

FIGURE 10: Local backward main path analysis of blockchain research.

the problem of blockchain applications in the wireless mobile network.

In the second path, Wang et al. [45] presented the first paper. This article mainly introduced how the blockchain affects value creation in the LP environment, as a rising IT artifact. Hughes et al. [46] used the IS/IM lens to study blockchain and presented a comprehensive overview of key themes in blockchain development. Based on blockchain, supply chain, network theory, and technology acceptance model, Queiroz and Fosso Wamba [47] established a model on the basis of the classical unified theory of technology acceptance and use through the study of emerging literature. The above three pieces of literature have one thing in common, which is through the method of literature research. And then, the rest of the papers study the application of blockchain in the IoT. Just like Reyna et al. [48], based on this relationship, this paper investigated the challenges of blockchain in the application of IoT and analyzed how the blockchain could potentially improve IoT.

Through the local backward main path analysis, we may draw the following conclusions. The first one is studying blockchain is often to combine blockchain with other fields, such as IoT and mobile services. Second, there is much more research on blockchain than the previous article, and this article is just the tip of the iceberg.

5.2.3. Global Standard Main Path. The aforementioned works in the local backward main path are mainly about the generalization of blockchain research. It is different from the path in Figure 9 and has eleven new papers are identified in the global standard main path. The front of the path in Figure 11 is consistent with the local forward path.

The paper on this path focuses on the study of the application and future development trend of blockchain research. As an early study of the blockchain application and development trend of the article, Nofer et al. [49] made a detailed introduction to the application and development of blockchain research, including digital currency and smart contract, and this paper laid a certain foundation for later research. The following papers, such as that by Risius and Spohrer [50],
Hawlitschek et al. [51], and Casino et al. [52], have conducted research on the present blockchain technology. Also, various research directions are determined. The difference is that Hawlitschek et al. [51] also looks at the potential of blockchain technology to solve any problem in the sharing economy.

For the seven papers at the end, they covered the application of blockchain technology in different industries, including the gas industry [53], smart contract [54], emerging healthcare technologies [55], and food consumption and its safety [56]. These studies have laid a good research foundation for future scholars.

In the above local backward path analysis, we summarize the broad scope of knowledge development. The global standard main path also confirms this. The four ways of this paper are different, but they complement each other from different angles and deepen the overall understanding and general direction of scholars in this field.

5.2.4. Global Key-Route Main Path. Whether local forward path, local backward path, or global standard path is used, the route with the highest weight is not necessarily among them. Besides, some knowledge diffusion paths that have relatively low weights also have a huge impact in this field may be ignored. To ameliorate these drawbacks, the keyroute main path is come up by Liu and $\mathrm{Lu}$ [39] to present more details of a specific domain. The key-route main path in Figure 12 has a chosen size of 10 to demonstrate, and 32 papers have been identified.

From Figure 12, we can see that the upper left corner branch is the same as the global standard main path. And the lower-left corner branch is the same as the local forward main path. Therefore, this part is no longer analyzed. Three new papers, including Kshetri $[57,58]$ and Kamble et al. [59], appear in the lower right corner of the branch, all three papers mentioned the relationship between blockchain and supply chain network and the role of the IoT among them, and this is consistent with the research topic of other articles along this path.

In the upper right corner is a brand new path, mainly based on blockchain-Internet of Things research. This combination can lay the foundation for the development of new business 


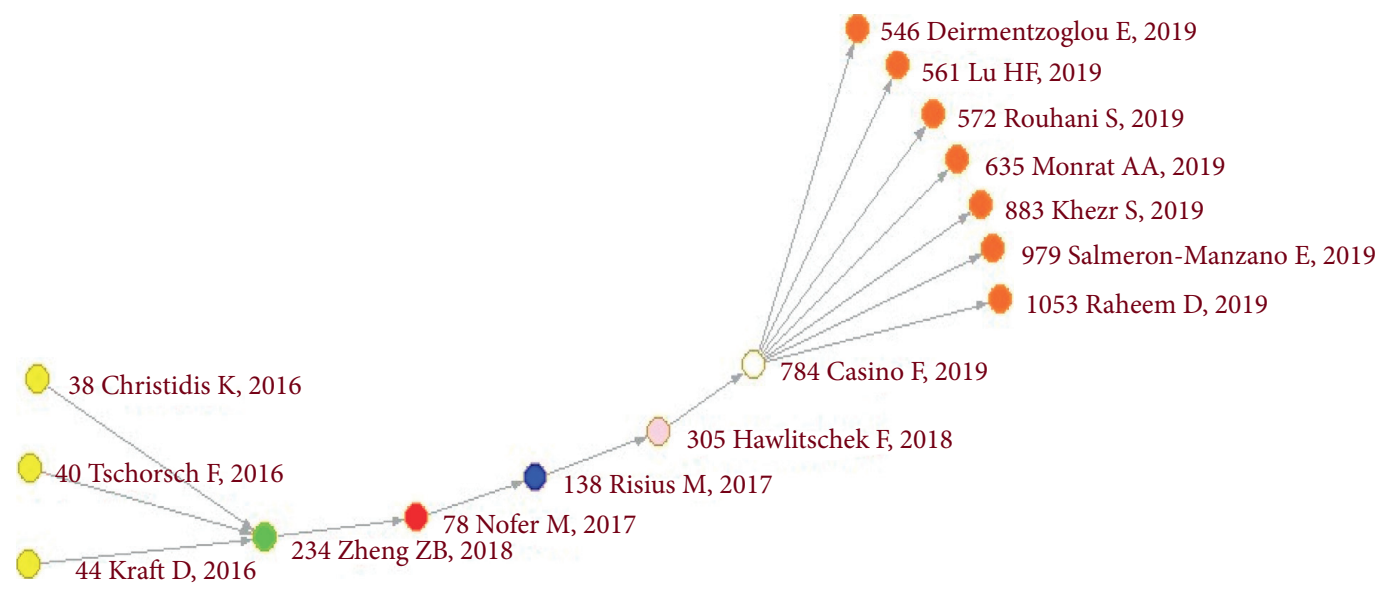

FIGURE 11: Global standard main path analysis of blockchain research.

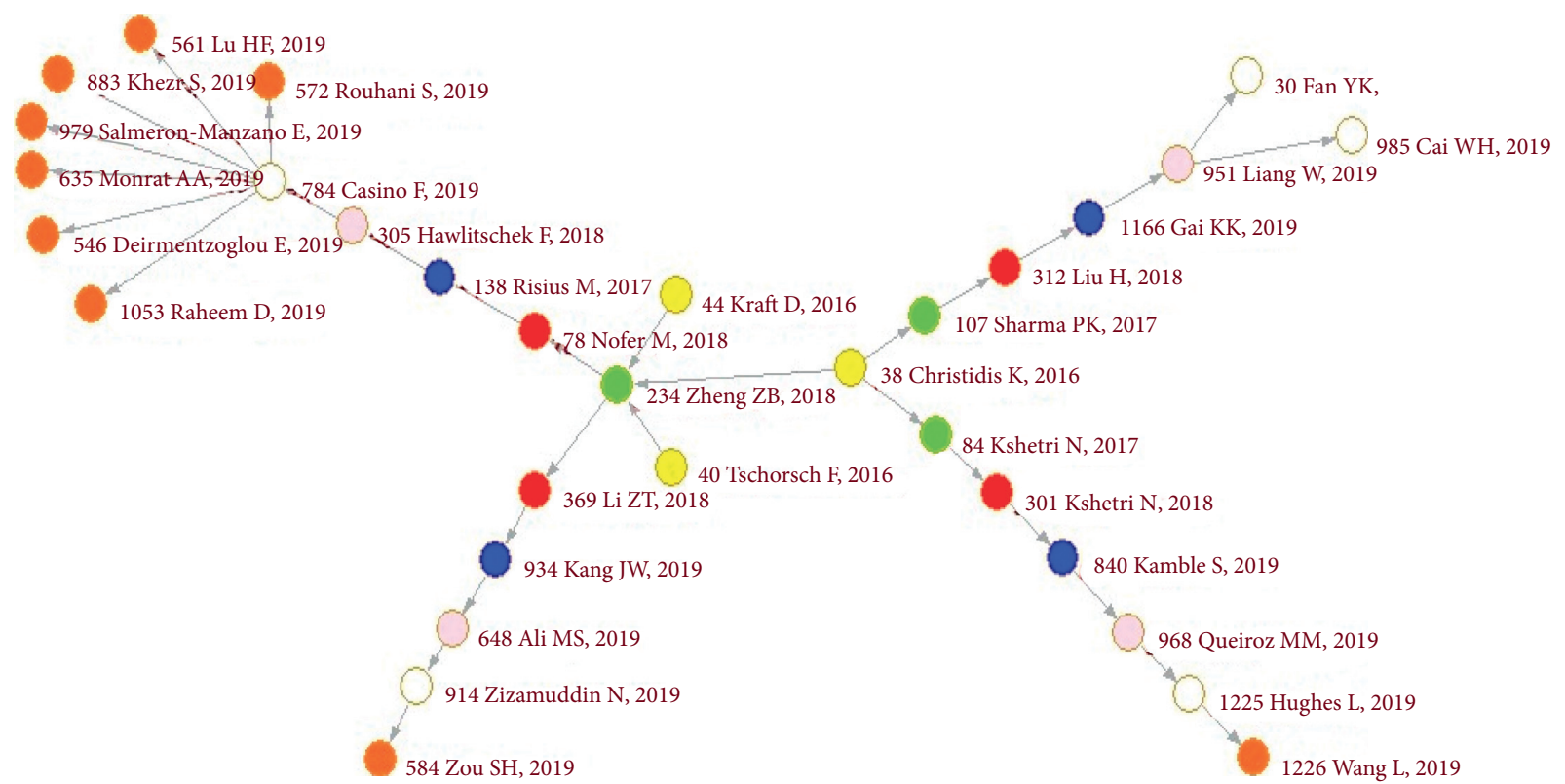

FIGURE 12: Global key-route main path analysis of blockchain research.

models and new types of distributed applications and can lead to major changes in multiple industries [3]. Many studies based on blockchain and the IoT are emerging. Sharma et al. [60] proposed a distributed and secure SDN architecture for the IoT, which used blockchain technology. Liang et al. [61] proposed a secure fabric data transmission technology based on blockchain for the industrial Internet of Things. Several other papers have mentioned the application of blockchain in various aspects and applied the IoT to it.

As can be seen from Figure 12, the development of blockchain research presents a $\pi$-type change trend and in the process of development has formed four different paths. It should be considered that, in the entire critical path diagram, we slightly expanded the path size, which resulted in almost covering the three main paths of the above, and this also visually reflected that the previous three-path analysis is more critical and also a knowledge dissemination structure that roughly covers the entire field. Through the analysis of these four paths, we find that the influence of top scholars in this field is very important. The Christidis, Tschorsch, and Kraft teams, for example, are at important juncture points in each of these main path structures, and have made significant contributions in terms of both quantity and quality of published papers.

From the analysis of the future development trend and main path of blockchain, it can be seen that the application of blockchain is mainly concentrated in digital cryptocurrency, financial trading and clearing, smart contract, corporate governance, and IoT. Few studies discuss the problems and risks existing in the development and maturity of blockchain and put forward corresponding solutions.

\section{Conclusions}

This paper has summarized the current status and emerging trends of blockchain research, which is helpful for beginners 
who are interested in blockchain to directly understand its development trend in recent years. Besides, we also have given the main path analysis of blockchain development to clarify the context of blockchain research.

The research results of this paper are summarized as follows: first, in the last five years, especially in the last two years, the number of research results on the blockchain has been increasing, indicating that blockchain has attracted more and more attention from scholars around the world. Second, China is the most active country in this field. The most prolific authors are not from China, whereas blockchain research is still booming in this country. Moreover, most of the authors and institutions active in the blockchain research field also have a significant influence on the research field. Thirdly, IEEE Access is the most productive journal in blockchain research. Engineering and Telecommunications, due to numerous research achievements, has also made a significant contribution to the development of the blockchain field. Given the hotspots and emerging trends in the field, "Bitcoin" is the keyword most frequently used, and the reference of Antonopoulos' has the strongest strength. Moreover, Merkle and Antonopoulos, as the frequently cited authors, have the longest duration that began in 2016 and ended in 2019 years. Lastly, we can see that, from the main path analysis results, the development of blockchain research has gone through a gradual process. The smart contract, IoT, and digital currency are all important nodes in the development of blockchain, and we also found that the development of blockchain research presents a $\pi$-type change trend. We provide a brief view for beginners who want to learn blockchain research directly and contribute to extend the theoretical foundation of blockchain research by giving a comprehensive bibliometric analysis. However, there are still some limitations in this research; for example, we mainly focus on analyzing blockchain research from a general perspective since it has been widely applied in various fields. However, this paper did not focus on blockchain research in a specific field such as finance or medical systems. As a result, deep insight into a certain area related to the blockchain would be our future research direction.

\section{Data Availability}

The literature data used to support the results of this study came from the Web of Science database.

\section{Ethical Approval}

This article does not contain any studies with human participants or animals performed by any of the authors.

\section{Conflicts of Interest}

The authors declare that they have no conflicts of interest.

\section{Authors' Contributions}

Sun Meng contributed to the conception of the study and also significantly to analysis and manuscript preparation.
Yan Chen performed the data analyses and wrote the manuscript. Wei Zhou updated the data and made revisions about the corresponding analytic results. Also, he rechecked the revised manuscript, ensuring all reviewers' concerns have been addressed. Dejian Yu helped in analysis with constructive discussion.

\section{Acknowledgments}

This work was supported by the Humanity and Social Science Youth Foundation of the Ministry of Education of China (no. 18YJC900118).

\section{References}

[1] C. R. Harvey, "Cryptofinance," 2015, https://ssrn.com/ abstract $=2438299$.

[2] L. Lee, "New kids on the blockchain: how bitcoin's technology could reinvent the stock market," Social Science Research Network Electronic Journal, vol. 12, no. 2, p. 81, 2016.

[3] K. Christidis and M. Devetsikiotis, "Blockchains and smart contracts for the internet of things," IEEE Access, vol. 4, pp. 2292-2303, 2016.

[4] D. Yermack, "Corporate governance and blockchains," Review of Finance, vol. 21, no. 1, pp. 7-31, 2017.

[5] D. Yu, "A scientometrics review on aggregation operator research," Scientometrics, vol. 105, no. 1, pp. 115-133, 2015.

[6] J. Song, H. Zhang, and W. Dong, "A review of emerging trends in global PPP research: analysis and visualization," Scientometrics, vol. 107, no. 3, pp. 1111-1147, 2016.

[7] Y. Chen and C. Wu, "The hot spot transformation in the research evolution of maker," Scientometrics, vol. 113, no. 3, pp. 1307-1324, 2017.

[8] T. Cui and J. Zhang, "Bibliometric and review of the research on circular economy through the evolution of Chinese public policy," Scientometrics, vol. 116, no. 2, pp. 1013-1037, 2018.

[9] C. Olmeda-Gómez, C. Romá-Mateo, and M.-A. Ovalle-Perandones, "Overview of trends in global epigenetic research (2009-2017)," Scientometrics, vol. 119, no. 3, pp. 1545-1574, 2019.

[10] R. Ma, Q. Dai, C. Ni, and X. Li, “An author co-citation analysis of information science in China with Chinese Google Scholar search engine, 2004-2006," Scientometrics, vol. 81, no. 1, pp. 33-46, 2009.

[11] M. Kozak, L. Bornmann, and L. Leydesdorff, "How have the Eastern European countries of the former Warsaw Pact developed since 1990? A bibliometric study," Scientometrics, vol. 102, no. 2, pp. 1101-1117, 2014.

[12] M. Swan, "Blockchain thinking: the brain as a decentralized autonomous corporation [commentary]," IEEE Technology and Society Magazine, vol. 34, no. 4, pp. 41-52, 2015.

[13] G. Zyskind and O. Nathan, "Decentralizing privacy: using blockchain to protect personal data," in Proceedings of the 2015 IEEE Security and Privacy Workshops, pp. 180-184, IEEE, San Jose, CA, USA, May 2015.

[14] F. Tschorsch and B. Scheuermann, "Bitcoin and beyond: a technical survey on decentralized digital currencies," IEEE Communications Surveys \& Tutorials, vol. 18, no. 3, pp. 2084-2123, 2016.

[15] X. Yue, H. Wang, D. Jin, M. Li, and W. Jiang, "Healthcare data gateways: found healthcare intelligence on blockchain with novel privacy risk control," Journal of Medical Systems, vol. 40, no. 10, pp. 1-8, 2016. 
[16] A. Azaria, A. Ekblaw, T. Vieira, and A. Lippman, "Medrec: using blockchain for medical data access and permission management," in Proceedings of the 2016 2nd International Conference on Open and Big Data (OBD), pp. 25-30, IEEE, Vienna, Austria, August 2016.

[17] J. Yli-Huumo, D. Ko, S. Choi, S. Park, and K. Smolander, "Where is current research on blockchain technology?-a systematic review," PLoS One, vol. 11, no. 10, p. 822, 2016.

[18] A. Kosba, A. Miller, E. Shi, Z. Wen, and C. Papamanthou, "Hawk: the blockchain model of cryptography and privacypreserving smart contracts," in Proceedings of the 2016 IEEE Symposium on Security and Privacy (SP), pp. 839-858, IEEE, San Jose, CA, USA, May 2016.

[19] Z. Zheng, S. Xie, H. N. Dai, X. Chen, and H. Wang, "Blockchain challenges and opportunities: a survey," International Journal of Web and Grid Services, vol. 14, no. 4, pp. 352-375, 2018.

[20] N. Z. Aitzhan and D. Svetinovic, "Security and privacy in decentralized energy trading through multi-signatures, blockchain and anonymous messaging streams," IEEE Transactions on Dependable and Secure Computing, vol. 15, no. 5, pp. 840-852, 2018.

[21] A. Dorri, M. Steger, S. S. Kanhere, and R. Jurdak, "Blockchain: a distributed solution to automotive security and privacy," IEEE Communications Magazine, vol. 55, no. 12, pp. 119-125, 2017.

[22] J. Kang, R. Yu, X. Huang, S. Maharjan, Y. Zhang, and E. Hossain, "Enabling localized peer-to-peer electricity trading among plug-in hybrid electric vehicles using consortium blockchains," IEEE Transactions on Industrial Informatics, vol. 13, no. 6, pp. 3154-3164, 2017.

[23] T.-T. Kuo, H.-E. Kim, and L. Ohno-Machado, "Blockchain distributed ledger technologies for biomedical and health care applications," Journal of the American Medical Informatics Association, vol. 24, no. 6, pp. 1211-1220, 2017.

[24] S. Underwood, "Blockchain beyond bitcoin," Communications of the ACM, vol. 59, no. 11, pp. 15-17, 2016.

[25] G. Wood, "Ethereum: a secure decentralised generalised transaction ledger," Ethereum Project Yellow Paper, vol. 151, pp. 1-32, 2014.

[26] D. Tapscott and A. Tapscott, "How blockchain will change organizations," MIT Sloan Management Review, vol. 58, no. 2, pp. 10-13, 2017.

[27] Z. Li, A. V. Barenji, and G. Q. Huang, "Toward a blockchain cloud manufacturing system as a peer to peer distributed network platform," Robotics and Computer-Integrated Manufacturing, vol. 54, pp. 133-144, 2018.

[28] M. A. Khan and K. Salah, "IoT security: review, blockchain solutions, and open challenges," Future Generation Computer Systems, vol. 82, pp. 395-411, 2018.

[29] I. Eyal, "Blockchain technology: transforming libertarian cryptocurrency dreams to finance and banking realities," Computer, vol. 50, no. 9, pp. 38-49, 2017.

[30] O. Novo, "Blockchain meets IoT: an architecture for scalable access management in IoT," IEEE Internet of Things Journal, vol. 5, no. 2, pp. 1184-1195, 2018.

[31] C. Chen, Z. Hu, S. Liu, and H. Tseng, "Emerging trends in regenerative medicine: a scientometric analysis inCiteSpace," Expert Opinion on Biological Therapy, vol. 12, no. 5, pp. 593-608, 2012.

[32] M. Pilkington, "Blockchain technology: principles and applications," Research Handbook on Digital Transformations, pp. 225-253, 2016.
[33] Y. Yuan and F. Y. Wang, "Blockchain: the state of the art and future trends," Acta Automatica Sinica, vol. 42, no. 4, pp. 481-494, 2016.

[34] M. S. Ali, M. Vecchio, M. Pincheira, K. Dolui, F. Antonelli, and M. H. Rehmani, "Applications of blockchains in the Internet of Things: a comprehensive survey," IEEE Communications Surveys \& Tutorials, vol. 21, no. 2, pp. 1676-1717, 2018.

[35] D. Kraft, "Difficulty control for blockchain-based consensus systems," Peer-to-Peer Networking and Applications, vol. 9, no. 2, pp. 397-413, 2015.

[36] J. Li, "Data transmission scheme considering node failure for blockchain," Wireless Personal Communications, vol. 103, no. 1, pp. 179-194, 2018.

[37] J. Kang, R. Yu, X. Huang et al., "Blockchain for secure and efficient data sharing in vehicular edge computing and networks," IEEE Internet of Things Journal, vol. 6, no. 3, pp. 4660-4670, 2019.

[38] N. Nizamuddin, K. Salah, M. Ajmal Azad, J. Arshad, and M. H. Rehman, "Decentralized document version control using ethereum blockchain and IPFS," Computers \& Electrical Engineering, vol. 76, pp. 183-197, 2019.

[39] J. S. Liu and L. Y. Y. Lu, "An integrated approach for main path analysis: development of the Hirsch index as an example," Journal of the American Society for Information Science and Technology, vol. 63, no. 3, pp. 528-542, 2011.

[40] S. Kim, "Two-phase cooperative bargaining game approach for shard-based blockchain consensus scheme," IEEE Access, vol. 7, pp. 127772-127780, 2019.

[41] Z. Liu, N. C. Luong, W. Wang et al., "A survey on blockchain: a game theoretical perspective," IEEE Access, vol. 7, pp. 47615-47643, 2019.

[42] L. Wang, X. Luo, and F. Lee, "Unveiling the interplay between blockchain and loyalty program participation: a qualitative approach based on Bubichain," International Journal of Information Management, vol. 49, pp. 397-410, 2019.

[43] H. Liu, Y. Zhang, and T. Yang, "Blockchain-enabled security in electric vehicles cloud and edge computing," IEEE Network, vol. 32, no. 3, pp. 78-83, 2018.

[44] Z. Xiong, Y. Zhang, D. Niyato, P. Wang, and Z. Han, "When mobile blockchain meets edge computing," IEEE Communications Magazine, vol. 56, no. 8, pp. 33-39, 2018.

[45] S. Wang, L. Ouyang, Y. Yuan, X. Ni, X. Han, and F. Y. Wang, "Blockchain-enabled smart contracts: architecture, applications, and future trends," IEEE Transactions on Systems, Man, and Cybernetics: Systems, vol. 49, no. 11, pp. 2266-2277, 2019.

[46] L. Hughes, Y. K. Dwivedi, S. K. Misra, N. P. Rana, V. Raghavan, and V. Akella, "Blockchain research, practice and policy: applications, benefits, limitations, emerging research themes and research agenda," International Journal of Information Management, vol. 49, pp. 114-129, 2019.

[47] M. M. Queiroz and S. Fosso Wamba, "Blockchain adoption challenges in supply chain: an empirical investigation of the main drivers in India and the USA," International Journal of Information Management, vol. 46, pp. 70-82, 2019.

[48] A. Reyna, C. Martín, J. Chen, E. Soler, and M. Díaz, "On blockchain and its integration with IoT: challenges and opportunities," Future Generation Computer Systems, vol. 88, pp. 173-190, 2018.

[49] M. Nofer, P. Gomber, O. Hinz, and D. Schiereck, "Blockchain," Business \& Information Systems Engineering, vol. 59, no. 3, pp. 183-187, 2017. 
[50] M. Risius and K. Spohrer, "A blockchain research framework," Business \& Information Systems Engineering, vol. 59, no. 6, pp. 385-409, 2017.

[51] F. Hawlitschek, B. Notheisen, and T. Teubner, "The limits of trust-free systems: a literature review on blockchain technology and trust in the sharing economy," Electronic Commerce Research and Applications, vol. 29, pp. 50-63, 2018.

[52] F. Casino, T. K. Dasaklis, and C. Patsakis, "A systematic literature review of blockchain-based applications: current status, classification and open issues," Telematics and Informatics, vol. 36, pp. 55-81, 2019.

[53] H. Lu, K. Huang, M. Azimi, and L. Guo, "Blockchain technology in the oil and gas industry: a review of applications, opportunities, challenges, and risks," IEEE Access, vol. 7, pp. 41426-41444, 2019.

[54] S. Rouhani and R. Deters, "Security, performance, and applications of smart contracts: a systematic survey," IEEE Access, vol. 7, pp. 50759-50779, 2019.

[55] S. Khezr, M. Moniruzzaman, A. Yassine, and R. Benlamri, "Blockchain technology in healthcare: a comprehensive review and directions for future research," Applied Sciences, vol. 9, no. 9, p. 1736, 2019.

[56] D. Raheem, M. Shishaev, and V. Dikovitsky, "Food system digitalization as a means to promote food and nutrition security in the barents region," Agriculture, vol. 9, no. 8, p. 168, 2019.

[57] N. Kshetri, "Can blockchain strengthen the internet of things?" IT Professional, vol. 19, no. 4, pp. 68-72, 2017.

[58] N. Kshetri, “Blockchain's roles in meeting key supply chain management objectives," International Journal of Information Management, vol. 39, pp. 80-89, 2018.

[59] S. Kamble, A. Gunasekaran, and H. Arha, "Understanding the blockchain technology adoption in supply chains-Indian context," International Journal of Production Research, vol. 57, no. 7, pp. 2009-2033, 2018.

[60] P. K. Sharma, S. Singh, Y.-S. Jeong, and J. H. Park, "DistBlockNet: a distributed blockchains-based secure SDN architecture for IoT networks," IEEE Communications Magazine, vol. 55, no. 9, pp. 78-85, 2017.

[61] W. Liang, M. Tang, J. Long, X. Peng, J. Xu, and K.-C. Li, “A secure FaBric blockchain-based data transmission technique for industrial internet-of-things," IEEE Transactions on Industrial Informatics, vol. 15, no. 6, pp. 3582-3592, 2019. 